\title{
A Study on the Antecedents and Consequences of Smart Plant Construction
}

\author{
Wonjong Kim ${ }^{1}$, Hyun-chul Jang ${ }^{2}$, Battumur Gerelmaa ${ }^{3}$, Gantumur Khongorzul ${ }^{4}$ \\ ${ }^{1}$ Professor, Department of Industrial Management, Gyeongsang National University, \\ BERI, Korea \\ ${ }^{2}$ Ph.D, Department of Industrial Management, Gyeongsang National University, \\ Korea \\ ${ }^{3}$ Ph.D student, Department of Industrial Management, Gyeongsang National \\ University, Korea \\ ${ }^{4}$ Lecturer, Department of Industrial Management, Gyeongsang National University, \\ Korea \\ 1'wj.kim@gnu.ac.kr, ${ }^{2} j h c @ u a t v a l v e . c o . k r,{ }^{3}$ battumurgerelmaa665@gmail.com, \\ ${ }^{4}$ khongorzul@gnu.ac.kr
}

\begin{abstract}
This study focuses on the success factor for Smart Plant construction. In this line, the exogenous variables that would affect expectations for competitiveness were hired from the related studies, including organizational factors, technical factors, and environmental factors. Moreover, the organizational factors have been divided into two sub-dimensions, i.e., the leadership of top management and competency development of organizational members, and the technical factors also have been divided into two sub-dimensions, i.e., technology relevance, safety, and risk-taking. The environmental factors consist of government support and influence of business partners. For the empirical analysis, a survey has been conducted. To examine the research model, survey data from employees of the related field were analyzed using confirmatory factor analysis and structural equation modeling techniques. The results of this study indicate that 1)Competency development of organizational members, technology relevance, and government support has a significant influence on the expectations for competitiveness enhancement, 2)Leadership of top management, Safety and risk-taking, and influence of business partners have not a significant influence on the expectations for competitiveness enhancement, 3)Expectations for competitiveness enhancement has shown a significant impact on Smart Plants construction, which proves that expectations for effectiveness can effectively enhance Smart Plant construction. This result is in the same context as previous studies.
\end{abstract}

Keywords: Organizational factor, Technical factor, Environmental factor, Expectations for competitiveness enhancement, Smart plant construction

\section{Introduction}

The Fourth Industrial Revolution brought about fundamental changes in the economy and industry globally. At the center of the wave, prominent countries are struggling hard to find

Article history:

Received (December 28, 2020), Review Result (January 30, 2021), Accepted (March 8, 2021) 
competitive strategies, for instance, 'Industry 4.0' in Germany, 'Advanced Manufacturing Partnership (AMP)' in the US, and 'Manufacturing Industry 2025' in China. Also, numerous countries are striving to stabilize their industrial competitiveness through industrial policies. South Korea is formulating policies such as the establishment of the Fourth Industrial Revolution Committee [1]. In Europe, the concept of Industry 4.0 is undergoing innovative modifications, such as building smart factories that can integrate information and communication technologies with traditional industries such as manufacturing. Representative technologies in Industry 4.0 include the Internet of Things (IoT), big data, and artificial intelligence [2]. For domestic companies to actively respond to these changes, it is necessary to review the introduction cases of advanced European companies in detail and establish a longterm strategy based on this.

Regarding smart factories, in the age of Industry 4.0 [3][4][5][6], the fourth industrial revolution and smart plants [7][8][9], smart factories of small and medium-sized enterprises (SMEs) [10], previous research on smart factories, and performance was analyzed. Several works have been done on technologies [11], and the applications of these technologies do not constitute a digital enterprise. It can be seen that the progress of the company must not only consider the introduction of technology at the same time but also should consider organizational and technical resources and environmental factors.

According to the limitations of previous research, the necessity for research in a new aspect is raised as follows. First, it is necessary to classify and organize common concepts and characteristics for smart factories and smart plants. Second, it is necessary to deviate from the limitations of previous studies that mainly deal with the technical aspect and to take a comprehensive approach that considers the organizational aspect and the environmental aspect from the management aspect. Third, research is needed to empirically verify the impact on the competitiveness of companies while identifying key success factors for a successful introduction of smart plant construction from the perspective of including organizational, environmental and technical aspects.

The purpose of this study is to focus the world's attention on a new paradigm that has recently been emerged as a major issue, namely, the 4th industrial revolution and the new economic growth center on the manufacturing industry. Also, it demonstrates the key success factors for smart plant construction: organizational factors (leadership of top management, competency development of organizational members), technical factors (technology relevance, safety, and risk-taking), environmental factors (government support, the influence of business partners) and the relationship among the above factors with expectations for competitiveness enhancement, and smart plant construction have been tested through structural equation model analysis.

\section{Theoretical background}

\subsection{Fourth industrial revolution}

Industry 4.0' is a motto that German public, private, and academics aimed at innovation in the manufacturing industry since 2011, and it have become a common concept that the Fourth Industrial Revolution is widely known to the public. In other words, the Fourth industrial revolution is based on hyper-connectivity, which is triggered by digital technologies such as artificial intelligence and big data. With the development of the Industrial Internet, the integration of connectivity and a new level of high-tech industrial system is playing a pivotal role to become a key catalyst for the fourth industrial revolution [12]. 


\subsection{Success factors for smart plant}

Plant is used in the same term as industrial equipment, and the plant industry includes the equipment industry for machinery and equipment for manufacturing and processing raw materials, and the software industry for engineering and construction, supervision, commissioning, and operation to install it. This means a compound industry [1]. In other words, it is also a process equipment industry that processes products that can be used or absorbed by humans after separating and refining necessary substances by reacting resources, water, trees, gas, oil, sun, and wind that exist in natural ecosystems. This means a compound industry [1]. Another definition can be said to be a series of activities in which the results of performing in the process targeting a system in which facilities, people, materials, machines, etc. are integrated, are realized in an optimal form for a purpose [7].

\subsubsection{Organizational factors}

The role of the CEO is to determine the vision, strategy, and policy, and lead the organization to grow and survive, including decision-making and problem-solving. Moreover, the more important role should be to motivate all members to participate in the management process, and in this process, should be actively supported in all aspects including resources and communication [13].

H1a: Leadership of top management will have a positive impact on the Expectations for competitiveness enhancement.

H1b: The competency development of organizational members will have a positive impact on the Expectations for competitiveness enhancement.

\subsubsection{Technical factors}

Goodhue [14] defines task technical suitability (TTF) as an appropriate level of business requirements and personal capabilities and defines the suitability of work and technology. The Task-Technical Fit Model refers to the degree of support of information technology when individuals perform tasks.

H1c: The technology relevance will have a positive impact on the Expectations for competitiveness enhancement.

H1d: Safety and risk-taking will have a positive impact on the Expectations for competitiveness enhancement.

\subsubsection{Environmental factors}

According to Song Jong-guk and Kim Hyuk-joon [15], government support is a typical means for the government to intervene in market activities. It is common to provide Research and Development (R\&D) subsidies or incentives to corporate R\&D investment through tax cuts, Yoo Chun and Kim Hak-min [16]. It has also been confirmed through research that companies that have received government support have positive effects such as increase in R\&D intensity and increase in sales. Noh Yong-Hwan [17] concluded that the government's R\&D support policy had a significant effect on patent applications and registrations of participating companies, but had shown no significant effect on company sales and operating profit.

H1e: Government support will have a positive impact on the Expectations for competitiveness enhancement. 
H1f: The influence of business partners will have a positive impact on the Expectations for competitiveness enhancement.

\subsection{Expectations for competitiveness enhancement}

Research on the establishment of smart factories by Park Jong-Sik and Jiang Jing-Sik, [18], the concept of efficient manufacturing of personalized products and related production and manufacturing technology research trends was studied, and ICT technology and existing product manufacturing based on Korea's smart factory. It was emphasized that it was necessary to enhance the value-added of the existing industries and initiate new industries related to smart factories through convergence technologies. Furthermore, it is necessary to establish a foundation for domestic competitiveness by systematically fostering smart factory-related industries and establishing a smart factory test bed for this, and that a smart factory that can be actually used in the field is needed by reflecting the continuous requirements of the actual manufacturing site in the policy.

In addition, it is necessary to establish a foundation for domestic competitiveness by systematically fostering smart factory-related industries and establishing a smart factory test bed for this, and that a smart factory that can be used in the field is needed by reflecting the continuous requirements of the manufacturing site in the policy.

$\mathrm{H} 2$ : Expectations for competitiveness enhancement will play a positive role in the Smart plant construction

\section{Research method}

\subsection{Research model}

This study focuses on the key success factor for Smart Plant construction. In this line, the exogenous variables that would affect expectations for competitiveness were gathered from the related studies, including organizational factors, technical factors, and environmental factors. Moreover, the organizational factors have been divided into two sub-dimensions, i.e., the leadership of top management and competency development of organizational members, and the technical factors also have been divided into two sub-dimensions, i.e., technology relevance, safety, and risk-taking. The environmental factors consist of government support and influence of business partners. The research model is shown in [Figure 1].

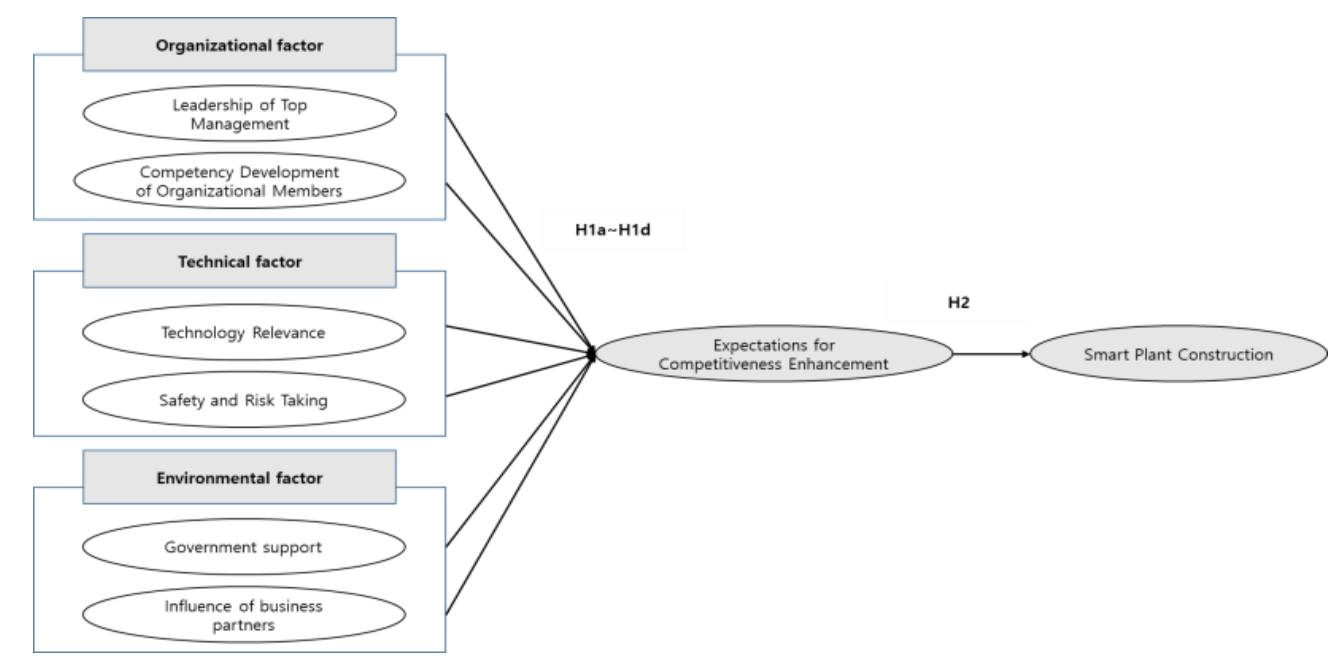


Figure 1. Research model

\subsection{Research methodology}

The analysis method of this study is as follows. First, confirmatory factor analysis and correlation analysis have been conducted to verify validity and reliability of the measurement. Second, to test the hypothesis, a path analysis based on the structural equation model was conducted to verify the established hypothesis.

In this study, the survey was conducted on entrepreneurs in large companies, where the analysis was performed using SPSS20.0 and AMOS18.0 for basic analysis and hypothesis testing. The data were collected for 1month from July 2020 to August 2020. A total of 146 copies of the questionnaire were collected, and the final 137 copies were utilized for analysis, including unfaithful responses. The main characteristics of the interviewees were $93.4 \%$ for men and $6.6 \%$ for women. In this age group, $52.6 \%$ of the $31-49$ age group, $25.5 \%$ of the 21 30 age group, and $21.9 \%$ of the age group over 50 . In terms of occupation, senior managers accounted for $38.7 \%$, and intermediate managers accounted for $29 \%$. Energy companies accounted for $54 \%$ of 74 companies, and EPC accounted for $20.4 \%$ of 28 companies.

Table 2. Confirmatory factor analysis

\begin{tabular}{|c|c|c|c|c|c|c|}
\hline & Estimate & S.E. & t-value & $\mathrm{p}$-value & C.R & AVE \\
\hline WM6 & .782 & & & & \multirow{6}{*}{0.875} & \multirow{6}{*}{0.621} \\
\hline WM5 & .819 & .095 & 10.297 & $* * *$ & & \\
\hline WM4 & .817 & .098 & 10.268 & $* * *$ & & \\
\hline WM3 & .791 & .100 & 9.863 & $* * *$ & & \\
\hline WM2 & .744 & .105 & 9.159 & $* * *$ & & \\
\hline WM1 & .775 & .110 & 9.622 & $* * *$ & & \\
\hline OD4 & .784 & & & & \multirow{4}{*}{0.904} & \multirow{4}{*}{0.703} \\
\hline OD3 & .895 & .095 & 11.651 & $* * *$ & & \\
\hline OD2 & .873 & .093 & 11.309 & $* * *$ & & \\
\hline OD1 & .797 & .093 & 10.075 & $* * *$ & & \\
\hline TR5 & .762 & & & & \multirow{5}{*}{0.859} & \multirow{5}{*}{0.549} \\
\hline TR4 & .712 & .115 & 8.322 & $* * *$ & & \\
\hline TR3 & .714 & .097 & 7.823 & $* * *$ & & \\
\hline TR2 & .768 & .101 & 8.387 & $* * *$ & & \\
\hline TR1 & .750 & .112 & 8.197 & $* * *$ & & \\
\hline SR4 & .847 & & & & \multirow{4}{*}{0.907} & \multirow{4}{*}{0.709} \\
\hline SR3 & .848 & .079 & 12.222 & $* * *$ & & \\
\hline SR2 & .896 & .089 & 13.265 & $* * *$ & & \\
\hline SR1 & .775 & .100 & 10.634 & $* * *$ & & \\
\hline GS4 & .928 & & & & \multirow{2}{*}{0.865} & \multirow{2}{*}{0.763} \\
\hline GS3 & .816 & .103 & 8.200 & $* * *$ & & \\
\hline IBP2 & .898 & & & & \multirow{2}{*}{0.838} & \multirow{2}{*}{0.723} \\
\hline IBP1 & .800 & .105 & 8.607 & $* * *$ & & \\
\hline EFE4 & .845 & & & & \multirow{3}{*}{0.868} & \multirow{3}{*}{0.622} \\
\hline EFE3 & .729 & .085 & 9.934 & $* * *$ & & \\
\hline EFE2 & .811 & .069 & 11.682 & $* * *$ & & \\
\hline
\end{tabular}




\begin{tabular}{|c|c|c|c|c|c|c|}
\hline EFE1 & .767 & .079 & 10.701 & $* * *$ & & \\
\hline SPI6 & .874 & & & & \multirow{6}{*}{0.891} & \multirow{6}{*}{0.696} \\
\hline SPC5 & .788 & .080 & 11.819 & $* * *$ & & \\
\hline SPC4 & .800 & .090 & 12.133 & $* * *$ & & \\
\hline SPC3 & .900 & .068 & 15.290 & $* * *$ & & \\
\hline SPC2 & .821 & .073 & 12.709 & $* * *$ & & \\
\hline SPC1 & .818 & .073 & 12.629 & $* * *$ & & \\
\hline
\end{tabular}

\section{Results}

\subsection{Confirmatory factor analysis}

The results are shown in [Table 2]. For models with goodness of fit to data: $\mathrm{CMIN}=613.678$, $\mathrm{CMIN} / \mathrm{df}=1.408, \quad \mathrm{CFI}=.951, \quad \mathrm{TLI}=.941, \quad \mathrm{IFI}=.952, \quad \mathrm{NFI}=.852, \quad \mathrm{GFI}=.808, \quad \mathrm{AGFI}=.753$ $\mathrm{RMR}=.028$, RMSEA=.055. According to the analysis, $\mathrm{CR}$ values were found to be $0.7(0.838 \sim 0.904)$ and AVE values over 0.5(0.549 0.723) for all variables. Therefore, the analysis could be reflected to have reliability and convergent validity.

\subsection{Correlation analysis}

The correlations among the variables were analyzed. As shown in [Table 3] the comparison of AVE square root and correlation. As a result of comparing the correlation of all two variables and the square root value of AVE, the correlation value is lower than the square root value of all AVE. Also, no pair of measures found with a correlation that exceeds 0.9, indicating no multi-co linearity exists among the construct. The correlation Matrix presented in [Table 3] supports all hypothesized positive relationships among the studied variables with high statistical significance.

Table 3. Correlation analysis

\begin{tabular}{|c|c|c|c|c|c|c|c|c|}
\hline & WM & OD & TR & $\mathrm{SR}$ & GS & IBP & EFE & SPC \\
\hline WM & .621 & & & & & & & \\
\hline OD & .341 & .703 & & & & & & \\
\hline TR & .357 & .311 & .549 & & & & & \\
\hline SR & .263 & .190 & .404 & .709 & & & & \\
\hline GS & .123 & .098 & .318 & .201 & .763 & & & \\
\hline IBP & .056 & .226 & .056 & .103 & .099 & .723 & & \\
\hline EFE & .253 & .605 & .162 & .122 & .209 & .336 & .622 & \\
\hline SPC & .236 & .691 & .248 & .164 & .256 & .263 & 619 & 696 \\
\hline
\end{tabular}

\subsection{Path analysis}

To test the hypothesis established in this study, covariance structural analysis was conducted and the results are shown in [Table 4]. For models with goodness of fit to data: CMIN=586.634, $\mathrm{CMIN} / \mathrm{df}=1.349, \quad \mathrm{CFI}=.958, \quad \mathrm{TLI}=.949, \quad \mathrm{IFI}=.959, \quad \mathrm{NFI}=.858, \quad \mathrm{GFI}=.819, \quad$ AGFI=.766 
$\mathrm{RMR}=.026, \mathrm{RMSEA}=.051$. The majority of indices show that they are above the baseline. The hypothesis test results are as follows.

Table 4. Path analysis

\begin{tabular}{|c|c|c|c|c|c|c|}
\hline & & Estimate & S.E. & C.R. & $\mathrm{P}$ & Results \\
\hline \multirow{2}{*}{$\begin{array}{c}\text { Organizational } \\
\text { Factor }\end{array}$} & H1a & .021 & .073 & .244 & .807 & Rejected \\
\hline & $\mathrm{H} 1 \mathrm{~b}$ & -.138 & .065 & 2.094 & .036 & Supported \\
\hline \multirow{2}{*}{$\begin{array}{l}\text { Technical } \\
\text { Factor }\end{array}$} & $\mathrm{H} 1 \mathrm{c}$ & .729 & .121 & 6.104 & $* * *$ & Supported \\
\hline & H1d & -.044 & .079 & -1.308 & .191 & Rejected \\
\hline \multirow{2}{*}{$\begin{array}{c}\text { Environmental } \\
\text { Factor }\end{array}$} & $\mathrm{H} 1 \mathrm{e}$ & .159 & .064 & 3.777 & $* * *$ & Supported \\
\hline & H1f & .303 & .060 & -.502 & .615 & Rejected \\
\hline \multicolumn{2}{|c|}{$\mathrm{H} 2$} & .973 & .098 & 9.758 & $* * *$ & Supported \\
\hline
\end{tabular}

\section{Conclusion}

This study aimed to identify the antecedents of smart plant construction mediating the expectation of competitiveness enhancement. The findings and implications are as follows.

First, it is possible to address the limitations of previous research on technology by positively looking at organizational factors, technical factors, and environmental factors that have a significant impact on the company's expectations for competitiveness. Furthermore, we can observe from the results that the expectation for competitiveness enhancement has a significant effect on the smart plant construction, the result that the company's competitiveness is the highest when organizational factors, environmental factors, and technical factors are all considered is emphasized in this study.

Second, among the key success factors of smart plants, the importance of the development of the impact of organization members is also emphasized. Therefore, to expect to strengthen corporate competitiveness, it is necessary to establish a clear vision, strategy, and plan for the smart plant based on the development of the influence of members of the organization, and to join and lead the change to the organization members by the introduction of new systems and technologies.

Third, the purpose of this technical factor is to secure data and the acquisition and processing of this data are major for business feasibility and engineering, equipment manufacturing and supply, construction and construction, operation, management and operation, and is the basis of the plant industry. Vulnerability to common core technologies such as value-added equipment, package equipment, management, and operation system, maintenance, and repair can be improved in more and more areas through securing and processing a lot of data, which is very much in the anticipation of strengthening corporate competitiveness. It will develop into an important factor.

Finally, the expectation of enhancing corporate competitiveness has a positive impact on the willingness to build smart factories. As discussed in the discussion above, the organizational factors, technical factors, and environmental factors set as the preceding variables in this study stimulate positive expectations for Smart Plant Construction, and it is confirmed that this affects the willingness to build smart plants.

The limitations of the study are as follows. First, the sample size is insufficient. Securing an appropriate sample of the object to be measured and the size of the sample is one of the key factors that have a great influence on the results of the study. However, since the empirical 
research related to smart plants is still in its infancy, there are many limitations in securing data at the individual level. In future research, efforts will be made to secure a large number of samples to derive clearer results. Second, technologies related to smart factories occupy a large number, but not all of them were covered. Future research will consider various technologies related to the smart factory so that more extensive research will be conducted than the current research.

\section{References}

[1] H. Ch. Jang, "The factors influencing smart plant construction and relationship with corporate competitiveness," Doctorial Dissertation, Gyeongsang National University, South Korea, (2021)

[2] Y. K. Lee, S. H. Chu, and H. Y. Meng, "European industry 4.0 status and introduction plans in energy plant field,” Korea Energy Engineering Association Conference, vol.229, no.1, (2019)

[3] J. P. Park, "Analysis on success cases of smart factory in Korea: Leveraging from large, medium, and small size enterprises," Journal of Digital Convergence, vol.15, no.5, pp.107-115, (2017)

[4] W. G. Oh, "The influence of the 4th industrial revolution on product lifecycle management: The perspective of the perception of expert groups," Doctorial Dissertation, Dongguk University, Korea, (2018)

[5] J. H. Yoon, "The fourth industrial revolution in South Korea: Discourse limitations and implications of response strategies," Public Policy Review, vol.32, no.1, pp.21-53, (2018)

[6] B. G. Cho and B. S. Yoon, "Current status of innovative companies and their support: Perspective of fourth industrial revolution,” Korea Business Association, vol.22, no.1, pp. 55-77, (2019)

[7] H. G. Pek and J. K. Lee, "In the era of the fourth industrial revolution, services drive manufacturing. The fourth industrial revolution and the domestic and foreign industries," Hyundai Economic Research Institute VIP Report, vol.706, no.17-33, pp.1-12, (2017)

[8] J. Yo, Ch. K. Yo, and Ch. H. Lee, "Fourth industrial and smart plant, NICE," vol.36, no.1, pp.26-44, (2018)

[9] S. G. Chin, Y. J. Lee, M. R. Kim, Ch. J. Lee, and M. G. Sang, "Training of mechanical equipment experts in the era of the fourth industrial revolution: Focusing on the mechanical equipment act and smart city," The Korean Association for Policy Studies, pp.1-20, (2018)

[10] S. M. Kim, "An empirical study on the smart factory acceptance intension and management performance of big data-based small and medium sized manufacturing companies," Doctorial Dissertation, Hansung University, South Korea, (2020)

[11] H. Ch. Kim, "An empirical study on adoption factor and performance analysis of smart factory through technical acceptance model - Focusing on TOE and IS success model," Doctorial dissertation, Hansung University, South Korea, (2019)

[12] P. C. Evans and M. Annunziata, "Industrial internet: Pushing the boundaries," General Electric Reports, (2012)

[13] P. Intakhan, "Direct and indirect effects of top management support on ABC implementation success: Evidence from ISO 9000 certified companies in Thailand," Procedia-Social and Behavioral Sciences, vol.164, pp.458470, (2014)

[14] D. L. Goodhue, "Understanding user evaluations of information systems," Management Science, vol.41, no.12, pp.1827-1844, (1995)

[15] J. G. Song and H. J. Kim, “The effectiveness of fiscal policies for R\&D,” Journal of Technology Innovation, vol.17, no.1, pp.1-48, (2009)

[16] Ch. Yo and H. M. Kim, "A study on the effectiveness of government's subsidy for SMEs R\&D activities," Korea Society of Trade Information, vol.16, no.5, pp.51-66, (2014)

[17] Y. H. Nyi, "Empirical researches, which SMEs are appropriate to be government backed?: The case of a Korean police loan program,” Entrepreneurship and Venture Research, vol.17, no.1, pp.1-17, (2014) 
[18] Ch. Sh. Park and G. Sh. Khang, "Strategies of smart factory building and application of small and mediumsized manufacturing enterprises," Journal of the Korea Safety management and science, vol.19.no.1, pp.227236, (2017) 